\title{
The effect of inhaling mother's breast milk odor on the behavioral responses to pain caused by hepatitis $B$ vaccine in preterm infants: a randomized clinical trial
}

Zahra Akbarian Rad ${ }^{1}$, Parvin Aziznejadroshan ${ }^{1 *}$ (D) Adeleh Saebi Amiri², Hemmat Gholinia Ahangar ${ }^{3}$ and Zahra Valizadehchari ${ }^{4}$

\begin{abstract}
Background: Nowadays, it is generally assumed that non-pharmacologic pain relief in preterm infants is an important measure to consider. Research findings suggest that familiar odors have soothing effects for neonates. The aim of this study was to compare the effect of maternal breast milk odor (MBMO) with that of another mother's breast milk odor (BMO) on the behavioral responses to pain caused by hepatitis $B(\mathrm{HB})$ vaccine injection in preterm infants.

Methods: This single-blind randomized clinical trial was performed over the period between February 2019 and March 2020 in the neonatal intensive care unit of Babol Rouhani Hospital, Iran. Ninety preterm infants, who were supposed to receive their $\mathrm{HB}$ vaccine, were randomly assigned into three groups: $M B M O(A)$, another mother's BMO $(\mathrm{B})$, and control with distilled water(C). Oxygen saturation ( $\mathrm{SaO} 2)$, blood pressure (BP) and heart rate (HR) were recorded for all participants through electronic monitoring. In addition, premature infant pain profiles (PIPP) were determined through video recording for all three groups during intervention. The chi-square, ANOVA and ANCOVA were used for analyzing the data, and $P<0.05$ was considered significant in this study.
\end{abstract}

Results: No significant differences were found between the three groups in mean \pm SD of HR, BP, and Sao2 before the intervention $(P>0.05)$. After the intervention, however, the means for heart rate in groups $A, B$, and $C$ were $146 \pm 14.3,153 \pm 17.5$ and $155 \pm 17.7$, respectively $(P=0.012)$. Moreover, the means for PIPP scores in groups $A, B$ and $C$ were $6.6 \pm 1.3,10 \pm 2$, and $11.4 \pm 1.9$, respectively $(P<0.001)$. There was no significant difference found between groups in their means of $\mathrm{SaO} 2$, systolic and diastolic blood pressure after the intervention $(P>0.05)$. Conclusions: The results indicate that stimulation with $\mathrm{MBMO}$ is effective in reducing pain in preterm infants; therefore, it can be postulated that this technique can be considered in less invasive procedures such as needling.

Trial registration: IRCT, IRCT20190220042771N1. Registered 18 May 2019- Retrospectively registered,

Keywords: Pain, Premature infant, Human milk, Odor, Hepatitis B vaccine

\footnotetext{
* Correspondence: aziznejadroshan@yahoo.com

'Non-Communicable Pediatric Disease Research Center, Health Research Institute, Babol University of Medical Sciences, Babol, I.R, Iran

Full list of author information is available at the end of the article
}

(c) The Author(s). 2021 Open Access This article is licensed under a Creative Commons Attribution 4.0 International License, which permits use, sharing, adaptation, distribution and reproduction in any medium or format, as long as you give appropriate credit to the original author(s) and the source, provide a link to the Creative Commons licence, and indicate if changes were made. The images or other third party material in this article are included in the article's Creative Commons. licence, unless indicated otherwise in a credit line to the material. If material is not included in the article's Creative Commons licence and your intended use is not permitted by statutory regulation or exceeds the permitted use, you will need to obtain permission directly from the copyright holder. To view a copy of this licence, visit http://creativecommons.org/licenses/by/4.0/ The Creative Commons Public Domain Dedication waiver (http://creativecommons.org/publicdomain/zero/1.0/) applies to the data made available in this article, unless otherwise stated in a credit line to the data. 


\section{Background}

Many innovative measures to relieve pain in preterm infants are considered by various neonatal intensive care units (NICU) worldwide $[1,2]$. It is assumed that neonatal pain in preterm infants can adversely affect their development in such multiple domains as nociceptive changes, altered brain development, stress systems, and functional abilities. Prolonged exposure to pain has also been associated with impaired brain development while preterm infants are in the NICU [3]. Pain assessment methods are currently performed through physiological (heart rate and respiratory rate) and behavioral criteria (crying time, changes in facial expression and limb movements) [4].

The premature infant pain profile (PIPP) is a set of measurable behavioral and physiological responses such as facial expression changes (squeezing eyes, raising eyebrows, wrinkling nasolabial groove) as well as changes in heart rate, $\mathrm{SaO} 2$, intrauterine age, and behavioral status of the infants, which are all definite reasons demonstrating pain in premature infants [5].

There is a strong tendency to use nonpharmacological interventions, as simple and secure techniques, for relieving pains in infants. Several methods have already been applied to relieve pain based on five senses [5]. Among them, the sense of smell is fully developed at birth [6] which can affect the neonate's emotional relationship with his/her mother [7]. Familiar odors, maternal odor for instance, supposedly have soothing effects on newborn infants. It is widely known that infants have the ability to detect their mother's breast odor even without experiencing breastfeeding at birth [8]. The breast milk odor (BMO) can enhance infants' sucking through the facial and trigeminal motor nerves in the brain, which, in turn, stabilizes the physiological state in infants [9]. In some cases, research findings has demonstrated that breastfeeding in human newborn infants can completely eliminates pain responses, and animal models have also depicted that the pain modulating effect of breastfeeding is likely mediated by opioid and non-opioid mechanisms [10]. Some studies have shown that Some other studies have also shown that fetal-maternal odors (mother's breast milk, body and amniotic fluid odors) can decrease stress responses including crying and motor activities in infants, especially those separated from breast milk or the ones under painful interventions [11]. In a relevant study, it was suggested that the maternal breast milk odor (MBMO) had a soothing effect on preterm infants, and that their pain score was lower than that of those exposed to formula odor [12]. Nevertheless, the results of Küçük Alemdar et al. (2017) demonstrated that the BMO made no statistically significant difference in the physiological and behavioral responses of MBMO group compared to other groups (amniotic fluid odor, maternal body odor and control groups) [13].

Given all the contradictory results on the effect of MBMO and another mother's BMO on preterm infants and the importance of pain relief for preterm infants, this study strove to investigate the effect of inhaling human milk on the behavioral responses of pain caused by $H B$ vaccine in preterm infants.

\section{Methods}

\section{Study design and setting}

This single-blind randomized clinical trial was done from February 2019 to March 2020 in a NICU of academic center (Rouhani Hospital, Babol, Iran).

\section{Participant}

Preterm infants 28-37 weeks of gestation, who have to be vaccinated for hepatitis B -zero turn the vaccinewere randomly assigned to three groups. The inclusion criteria were infants with no painful procedure and no feeding for up to $1 \mathrm{~h}$ before the intervention, stability in vital signs, no head and skull abnormalities as well as no receiving painkillers, sedatives and anticonvulsants. The exclusion criteria were maternal withdrawal from the study and infant sever disease or death.

\section{Groups characteristic}

After obtaining written consent from the parents, each of the eligible subjects was assigned a number. The numbers were written on paper and tossed into the box, and the desired number was taken out of the box by drawing lots based on the assigned rank. Statistics specialist generated the random allocation sequence, one of the researchers enrolled participants, and assigned infants to three groups: MBMO (A), another mother's BMO (B) and control with distilled water $(\mathrm{C})$. This study followed the CONSORT guidelines for reporting randomized controlled trials (Fig. 1).

\section{Sample size}

Considering $80 \%$ power and 0.05 error probability in this study, the number of cases was determined to be at least 30 neonates in each group [2].

\section{Data collection and processing}

According to the ward's schedule during the first 4 days of life, injection of $\mathrm{HB}$ vaccine was done. Preterm neonate was placed on a warmer by servo control and skin temperature $36.5-37^{\circ} \mathrm{C}$ in quiet room. All conditions including room temperature $\left(25^{\circ} \mathrm{C}\right)$, light, injection device were the same for all three groups as well as the vaccine administration was done by one person. The researcher and nurse did not use any aromatic substances in vaccination room during the study. The probe of 


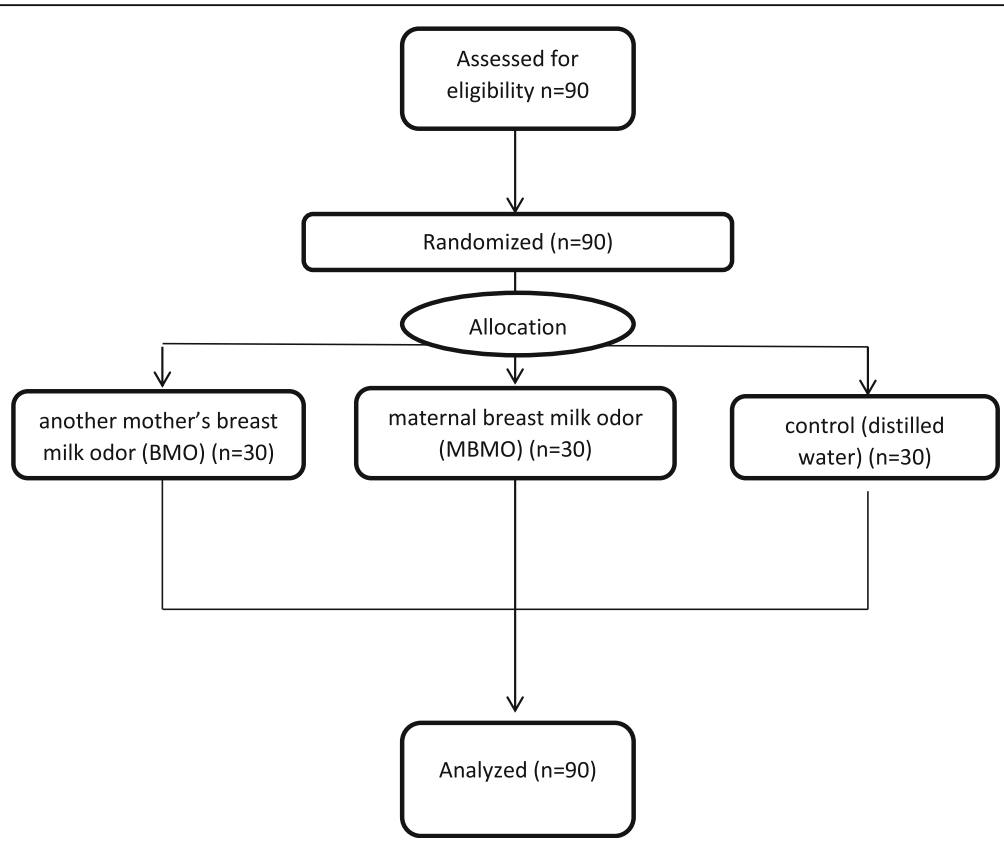

Fig. 1 study flowchart: allocation to study groups

monitoring was placed on the right wrist of baby without applying additional pressure. Heart rate, blood pressure and $\mathrm{SaO} 2$ of all preterm infants were recorded before starting the intervention as the initial time and immediately after completing vaccination (using the standard pulse oximeter and ECG monitoring of Saadat Company, Iran).

In both groups of $\mathrm{A}$ and $\mathrm{B}$, the breast milk samples taken in the early morning before eating breakfast were used to stimulate the smell sense of neonates. Pouring 2 $\mathrm{ml}$ of maternal breast milk and another mother's breast milk on a cotton swab was done as an intervention, and $2 \mathrm{ml}$ of distilled water as control group (group C). Next, these swabs were placed three centimeters away from the baby's nose. This process started $3 \mathrm{~min}$ before vaccination and continued until the vaccination was completed [2].

\section{Pain measurement}

The Premature Infant Pain Profile (PIPP) was used as the primary outcome variable. PIPP scores were recorded immediately before and after the vaccination for each infant. The PIPP is a behavioral measure of pain for premature infants. It includes seven indicators: 1 ) gestational age, 2) the behavioral state, 3) change in heart rate, 4) change in oxygen saturation, 5) brow bulge; 6) eyes squeeze and 7) nasolabial furrow. The total score is the summation of all seven indicators, with a minimum of 0 and maximum of 21 ; the higher the score, the greater the pain behavior [14]. If the overall PIPP score is between 0 and 6 points, the pain level is mild; if between 7 and 12 points, it is moderate; and if between 13 and 21 points, it is severe [15].

The PIPP tool was revised and validated for use with preterm babies born at 26-37 weeks of gestation by Gibbins et al. in 2014 [16].

Video recording of behavioral responses was taken from the beginning to the end of the process by a trained nurse, and then PIPP scoring was performed through watching video by the first author. The scoring was done while the video viewer was unaware of the test group. Throughout the intervention, any actions on the neonates such as contact, movement and so on were avoided.

Data were collected by using the demographic questionnaire including: birth weight, current disease (respiratory distress syndrome, transient tachypnea of newborn, sepsis and very low birth weight), sex, gestational age, postnatal age, Apgar score and PIPP score.

\section{Data analysis}

Statistics advisor performed the data analysis blindly by using SPSS Version 18. Descriptive information was shown as frequency, percentage, mean and standard deviation. Chi-square test for the relationship between two qualitative variables (demographic and PIPP qualitative variables with group variable), ANOVA test for comparing quantitative variables at the levels of more than two variables (quantitative demographic variables with group variable) and ANCOVA test for comparing research outcomes (SBP, DBP, $\mathrm{SaO} 2$ and heart rate) were used to 
remove the pretest effect and a $P$ value $<0.05$ were considered significant.

\section{Ethical consideration}

The study protocol was approved by the Ethics Committees of Babol University of Medical Sciences (IR.MUBABOL.REC.1397.253). The trial is registered in the IRCT20190220042771N1 Before participation in the study, written informed consent was obtained from each child's primary guardian.

\section{Results}

\section{Study subjects}

ALL 90 preterm infants, who included, were completed the study. The infants of the three groups were not significantly different in terms of sex, age, infant's current disease (Spsis, Respiratory Distress Syndrome (RDS), Transient Tachypnea of Newborn (TTN), very low birth weight (VLBW), gestational age, weight and APGAR score $(p>0.05)$ (Table 1).

Table 2 shows variables including SBP, DBP, $\mathrm{SaO} 2$ and heart rate before and after the intervention by using ANCOVA test.

As shown in Table 2, by eliminating the effect of the pretest variable and use of ANCOVA test, there is no significant difference between the means \pm SD of SBP $(p=0.482)$, DBP $(p=0.341)$ and $\mathrm{SaO} 2(p=0.193)$ in terms of group membership. ANCOVA test showed that change in heart rate was significantly lower in group A $(p=0.012)$ (Table 2).
PIPP score

The mean \pm SD of pain score in group A was $6.6 \pm 1.3$, and $10 \pm 2$ and $11.4 \pm 1.9$ in groups $B$ and $C$, respectively. The ANOVA test showed that there is a significant difference between groups $(P<0.001)$, and the results of post-hoc Tukey's test determined that this difference was between group A with groups B and C (Fig. 2).

\section{Discussion}

This study showed that MBMO greatly affected heart rate as well as behavioral responses to pain scoring in preterm infants compared with another mother's BMO and the control groups, but there were no significant differences found between the three groups in terms of SBP, DBP and $\mathrm{SaO} 2$.

Zhang et al. (2018) conducted a systematic review study to investigate the analgesic effects of BMO on infants. Their results demonstrated that there was a change in the heart rate of infants and that $\mathrm{SaO} 2$ pain scores were lower in MBMO group during and after blood sampling compared with those of the control group [17], It is worth noting that their result is consistent with the findings of the current study, except for $\mathrm{SaO} 2$. The stimulation with MBMO had a soothing effect on the behavioral responses to pain and reduced infant's pain in our study.

In another relevant study, Sajjadi et al. (2016) reported that the mean scores of PIPP had a significant effect on MBMO group compared to control group [2]. Nonetheless, a significant difference was found in the heart rate as well as $\mathrm{SaO} 2$ after the intervention. Their results are in line with those of the present study, except for change

Table 1 Comparison of demographic variables of preterm infants in three groups

\begin{tabular}{|c|c|c|c|c|}
\hline $\begin{array}{l}\text { Groups } \\
\text { Variable }\end{array}$ & $\operatorname{MBMO}(\mathrm{A})$ & $\begin{array}{l}\text { Another mother } \\
\text { BMO(B) }\end{array}$ & Control(C) & $\begin{array}{l}P \\
\text { value }\end{array}$ \\
\hline Sex n(\%) & & & & $0.562^{\mathrm{a}}$ \\
\hline Male & $15(50)$ & $16(53.3)$ & $12(40)$ & \\
\hline Female & $15(50)$ & $14(46.7)$ & $18(60)$ & \\
\hline Infant's age (hour) n(\%) & & & & $0.112^{\mathrm{a}}$ \\
\hline $24-48$ & $16(53.3)$ & $12(40)$ & $20(66.7)$ & \\
\hline $48-96$ & $14(46.7)$ & $18(60)$ & $10(33.3)$ & \\
\hline Infant's disease n(\%) & & & & $0.943^{\mathrm{a}}$ \\
\hline RDS, Sepsis & $3(10)$ & $4(13.3)$ & $3(10)$ & \\
\hline VLBW & $22(73.3)$ & $23(67.7)$ & $23(67.7)$ & \\
\hline TTN & $5(16.7)$ & $3(10)$ & $4(13.4)$ & \\
\hline $\begin{array}{l}\text { Infant's gestational age (WK) } \\
\text { (Mean } \pm \mathrm{SD})\end{array}$ & $32.9 \pm 2.4$ & $31.5 \pm 2.1$ & $32.5 \pm 2.4$ & $0.074^{\mathrm{b}}$ \\
\hline $\begin{array}{l}\text { Infant's weight }(\mathrm{g}) \\
(\text { Mean } \pm \mathrm{SD})\end{array}$ & $1806 \pm 553$ & $1620 \pm 425$ & $1688 \pm 404$ & $0.294^{b}$ \\
\hline Infant'sApgar score(Mean \pm SD) & $7.8 \pm 1$ & $7.6 \pm 1.3$ & $8.3 \pm 0.9$ & $0.071^{\mathrm{b}}$ \\
\hline
\end{tabular}

${ }^{a}$ Chi $^{2},{ }^{b}$ ANOVA 
Table 2 Mean and standard deviation scores of SBP, DBP, SaO2 and heart rate in the studied groups pre and post intervention

\begin{tabular}{|c|c|c|c|c|c|c|c|}
\hline \multirow{2}{*}{$\begin{array}{l}\text { Groups } \\
\text { Variables }\end{array}$} & \multicolumn{2}{|l|}{$\operatorname{MBMO}(\mathrm{A})$} & \multicolumn{2}{|c|}{ Another mother's BMO(B) } & \multicolumn{2}{|l|}{ Control(C) } & \multirow{2}{*}{$\begin{array}{l}P \\
\text { value }\end{array}$} \\
\hline & Pre intervention & Post intervention & Pre intervention & Post intervention & Pre intervention & $\begin{array}{l}\text { Post } \\
\text { intervention }\end{array}$ & \\
\hline $\mathrm{SBP}(\mathrm{mm} \mathrm{Hg})$ & $69.3 \pm 9.4$ & $70.9 \pm 8.2$ & $69.5 \pm 7.6$ & $70.2 \pm 6$ & $69.7 \pm 9$ & $71.7 \pm 9$ & 0.482 \\
\hline $\mathrm{DBP}(\mathrm{mm} \mathrm{Hg})$ & $40.6 \pm 9.8$ & $43.6 \pm 0.5$ & $40.8 \pm 9.9$ & $41.7 \pm 7.1$ & $40.9 \pm 7.9$ & $44 \pm 10.7$ & 0.341 \\
\hline $\mathrm{SaO} 2(\%)$ & $97 \pm 2.7$ & $95.2 \pm 5.2$ & $97.1 \pm 3.7$ & $94 \pm 6.2$ & $96.4 \pm 3.2$ & $91.1 \pm 11.7$ & 0.193 \\
\hline Heart rate & $139 \pm 16.1$ & $146 \pm 14.3$ & $141 \pm 15.6$ & $153 \pm 15.5$ & $139 \pm 17.8$ & $155 \pm 17.7$ & 0.012 \\
\hline
\end{tabular}

${ }^{a}$ ANCOVA test

in SaO2. Likewise, Küçük Alemdar et al. (2017) conducted a study through which they investigated the effect of mother's BMO, amniotic fluid odor, and body odor on the physiological and behavioral responses to heel stick pain in preterm infants and found no statistically significant difference between groups in terms of physiological and behavioral responses to pain such as heart rate, duration of crying and pain scale. Although the $\mathrm{SaO} 2$ was slightly different in the amniotic fluid odor group [13], As it can be seen, this finding is vividly inconsistent with the results of the present study. One possible reason for this discrepancy is the difference between both studies in terms of methodology and intervention process. In their study, $5 \mathrm{cc}$ of the mother's breast milk was poured on a sponge and placed five centimeters away from the neonate's nose for fifteen minutes before and after the intervention,, while the cotton swab had been placed three centimeters away from the infant's nose in our study. This process started $3 \mathrm{~min}$ before vaccination in the current study and continued until the vaccination was completed. Attempts were also made to minimize the effect of accustoming to the sense of smell in our study. Aziznejad et al. (2013) evaluated the physiological indicators and concluded that there was a statistically significant difference in the respiratory rate only between the intervention group with sucrose and the other groups immediately after the intervention, but there was no significant difference between the four groups in other variables (duration of crying, heart rate and $\mathrm{SaO} 2$ ) [18].

In three above-mentioned studies (Zhang, Sajjadi and Küçük Alemdar) which were different with our study in terms of methodology, there were no significant changes found in $\mathrm{SaO} 2$ between the intervention group and the control group. Moreover, in a similar study by Aziznejad et al. (2013), which was performed under the same condition, there were no differences in $\mathrm{SaO} 2$, either. One possible reason could be the difference in the equipment used.

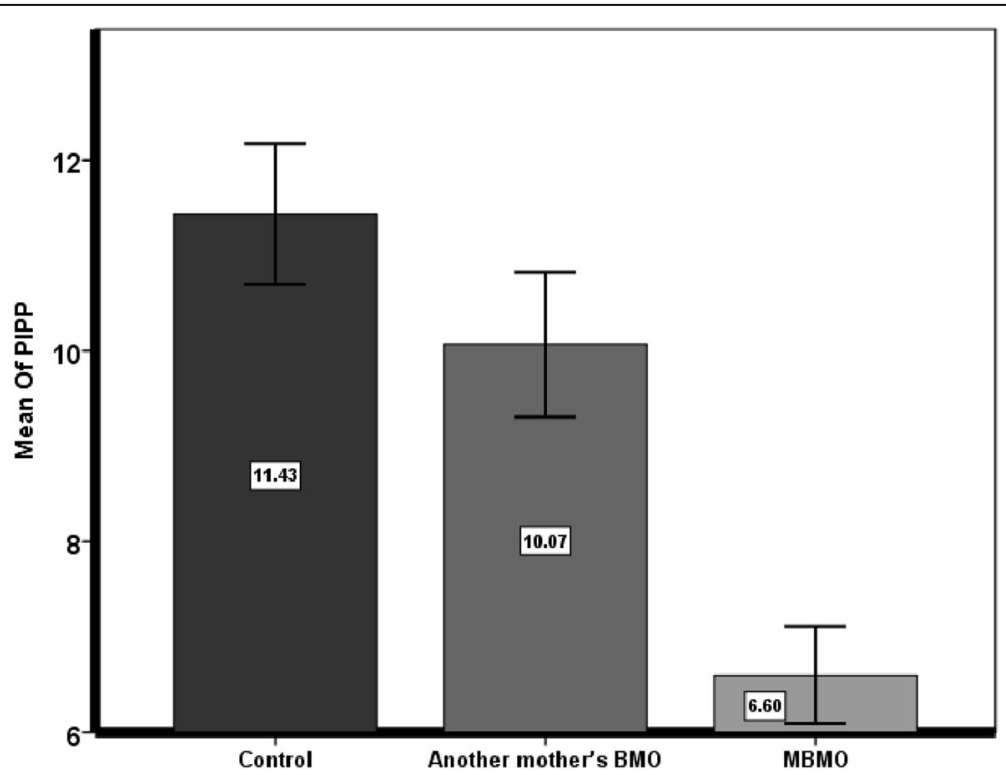

Fig. 2 The PIPP score's changes in three groups (Note: the same letters indicate no significant difference at level 5\%) 


\section{Conclusion}

On the basis of this research, the MBMO can be used as a familiar smell to manage the preterm infant's pain before performing any needling procedures such as vaccination.

\section{Limitations}

Due to the limited amount of equipment, the use of special probes for infants during the study was provided by several companies. The differences in the sensitivity of these probes may have caused the $\mathrm{SaO} 2$ changes not to be accurately determined.

\section{Abbreviations \\ ANOVA: Analysis of Variance; MBMO: Maternal Breast Milk Odor BMO: Another Mother's Breast Milk Odor; HBV: Hepatitis B Vaccine; HR: Heart Rate; NICU: Neonatal Intensive Care Unit; PIPP: Premature Infant Pain Profile; SBP: Systolic Blood Pressure; DBP: Diastolic Blood Pressure; SaO2: Arterial Oxygen Saturation; RDS: Respiratory Distress Syndrome; VLBW: Very low birth weight; TTN: Transient Tachypnea of Newborn; ANCOVA: Analysis of Covariance; chi²: Chi-square; APGAR: Appearance, Pulse, Grimace, Activity, Respiration}

\section{Acknowledgements}

The authors would like to thank the Research Deputy of Babol University of Medical Sciences, authorities, and colleagues in the Neonatal intensive care unit of Babol Rouhani Hospital as well as the infants' parents, who helped us throughout this study.

\section{Authors' contributions}

ASA studied the conception/design, carried out the analysis, interpreted the data, and helped drafting the manuscript. PA, the first supervisor of the study, studied the conception/design, analyzed the data, helped drafting the manuscript, did the critical revisions for important intellectual content, provided administrative/ technical/ material support, and did the final revision. ZAR critically revised the proposal, did the analysis, interpreted the data, and designed the article. The shares of the first and the third authors was equal. HGA performed the data analysis, and ZV contributed in the data collection. All authors read and approved the final manuscript.

\section{Funding}

This research received a grant from Babol University of Medical Sciences (Grant number: 258-9706616). The Babol university of Medical Sciences did not have any role in the design of the study, data collection, analysis, interpretation of the data, and the writing of the manuscript.

\section{Availability of data and materials}

The datasets analyzed in the current study are not publicly available due to an agreement with the participants upon the confidentiality of the data, but they are available from the corresponding author upon request.

\section{Ethics approval and consent to participate}

The study protocol was approved by the Ethics Committees of the Babol University of Medical Sciences (IR.MUBABOL.REC.1397.253). The trial is registered in the Iranian Registry of Clinical Trial (IRCT20190220042771N1). Before participation in the study, written informed consent was obtained from each child's primary guardian.

\section{Consent for publication}

The article does not contain any individual' details and consent for publication is not applicable.

\section{Competing interests}

The authors declare that they have no competing interests.

\section{Author details}

${ }^{1}$ Non-Communicable Pediatric Disease Research Center, Health Research

Institute, Babol University of Medical Sciences, Babol, I.R, Iran. ${ }^{2}$ Student
Research Committee, Babol University of Medical Sciences, Babol, I.R, Iran. ${ }^{3}$ Health Research Institute, Babol University of Medicine Sciences, Babol, I.R, Iran. ${ }^{4}$ Clinical Research development unit of Rohani hospital, Health Research Institute, Babol University of Medical Sciences, Babol, I.R, Iran.

Received: 3 August 2020 Accepted: 21 January 2021

Published online: 01 February 2021

References

1. Majidipour N, Nirouzad F, Sarrafzade S, Kalani L, Aghababaeian H, Haghighat BS. The effect of holy Quran recitation on the physiological responses of premature infants during phlebotomy: a randomized clinical trial. Int J Pediatr. 2018:6(7):7869-81.

2. Sajjadi M, Basirimoghadam M, Amiri SE. Effect of breast Milk odor on physiological and behavioral pain responses caused by hepatitis B vaccine in full-term infants. Horizon Med Sci. 2017;23(3):169-73.

3. Grunau RE. Long term effects of pain-related stress on neurodevelopment and pain perception of infants born very prematurely. Enfance. 2013;1:15-31.

4. Stadtlander L. Understanding Infant Pain. Int J Childbirth Educ. 2018;33(2):6-8.

5. Verklan MT, Walden M, Forest S. Core curriculum for neonatal intensive care nursing e-book: Elsevier health sciences; 2020.

6. Jebreili M, Rasouli AS, Hosseini MB, Gojazade M, Esfahlani HN. The effect of breast Milk odor in venipuncture pain in preterm infants: randomized controlled clinical trial. Majallah-i pizishki-i Danishgah-i Ulum-i Pizishki va Khadamat-i Bihdashti-i Darmani-i Tabriz. 2016;38(3):42.

7. Seraji A, Vakilian K. The comparison between the effects of aromatherapy with lavender and reathing techniques on the reduction of labor pain. Complement Med J. 2011;1(1):34-41.

8. Iranmanesh S, Shamsi A, Pour Aboli B, Movahedi Z. The effect of breast milk odor on transition time from gavage to oral feeding and hospital stay in premature infants. ICAN: Infant Child Adolesc Nutr. 2015;7(1):5-11.

9. Marofi M, Nikobakht F, Ali MN, Badiei Z. Comparing the effect of listening to melody vs. breast-feeding on neonates'pain intensity during heel-blood sampling in neonatal intensive care unit; 2015

10. Holsti L, Oberlander TF, Brant R. Does breastfeeding reduce acute procedural pain in preterm infants in the neonatal intensive care unit? A randomized clinical trial. Pain. 2011:152(11):2575-81.

11. Nishitani S, Miyamura T, Tagawa M, Sumi M, Takase R, Doi H, et al. The calming effect of a maternal breast milk odor on the human newborn infant. Neurosci Res. 2009;63(1):66-71

12. Badiee $Z$, Asghari M, Mohammadizadeh $M$. The calming effect of maternal breast milk odor on premature infants. Pediatr Neonatol. 2013;54(5):322-5.

13. Küçük Alemdar D, Kardaş ÖF. Effects of having preterm infants smell amniotic fluid, mother's milk, and mother's odor during heel stick procedure on pain, physiological parameters, and crying duration. Breastfeed Med. 2017;12(5):297-304.

14. Ranjbar A, Bernstein C, Shariat M, Ranjbar H. Comparison of facilitated tucking and oral dextrose in reducing the pain of heel stick in preterm infants: a randomized clinical trial. BMC Pediatr. 2020;20:1-9.

15. Stevens BJ, Gibbins S, Yamada J, Dionne K, Lee G, Johnston C, et al. The premature infant pain profile-revised (PIPP-R): initial validation and feasibility. Clin J Pain. 2014;30(3):238-43.

16. Gibbins S, Stevens BJ, Yamada J, Dionne K, Campbell-Yeo M, Lee G, et al. Validation of the premature infant pain profile-revised (PIPP-R). Early Hum Dev. 2014;90(4):189-93.

17. Zhang S, Su F, Li J, Chen W. The analgesic effects of maternal milk odor on newborns: a meta-analysis. Breastfeed Med. 2018;13(5):327-34.

18. Aziznejad P, Zahed Pasha Y, Ahmadpour Kacho M, Haji Ahmadi M, Mohammadzadeh I, Arzani A, et al. Comparing the effect of oral sucrose, breast milk and EMLA cream on acute pain during venipuncture in full term neonates. J Babol Univ Med Sci. 2013;15(3):16-23.

\section{Publisher's Note}

Springer Nature remains neutral with regard to jurisdictional claims in published maps and institutional affiliations. 